\title{
Expression of c-Met and hepatocyte growth factor in various gastric pathologies and its association with Helicobacter pylori infection
}

\author{
CHUAN XIE* ${ }^{*}$ ZHEN YANG* ${ }^{*}$ YI HU, XIMEI CAO, JIANG CHEN, YIN ZHU and NONGHUA LU
}

Department of Gastroenterology, The First Affiliated Hospital of Nanchang University, Nanchang, Jiangxi 330006, P.R. China

Received December 13, 2015; Accepted May 16, 2017

DOI: $10.3892 / 01.2017 .6983$

\begin{abstract}
Activation of the hepatocyte growth factor (HGF)/c-Met signaling pathway was identified to be associated with malignant tumors. The present study aimed at determining the expression of HGF and c-Met in gastric carcinogenesis and its association with Helicobacter pylori infection. Gastric biopsies were obtained from $160 \mathrm{H}$. pylori-negative and -positive patients, including those with chronic gastritis, intestinal metaplasia, dysplasia and gastric cancer (GC). Proteins were extracted from GES-1, gastric epithelial, and AGS, gastric adenocarcinoma, cells following co-culture with $H$. pylori in vitro. The expression of HGF and c-Met in gastric tissues or cells was determined using immunohistochemistry and western blot analysis. The expression of c-Met increased in GC tissues (72.5\%) compared with that in pre-cancerous lesions $(17.5,17.5$ and 30\%). Additional analysis identified that the expression of HGF and c-Met was significantly increased in the presence of $H$. pylori infection in dysplasia and gastric cancer samples. Furthermore, H. pylori may activate the $\mathrm{HGF} / \mathrm{c}-\mathrm{Met}$ signaling pathway in vitro, which may contribute to gastric carcinogenesis.
\end{abstract}

\section{Introduction}

Gastric cancer is the fourth most common type of malignancy, and the fourth and fifth leading cause of cancer-associated mortality in male and female worldwide, respectively, and the situation is poor in developing countries (1). The recognized etiology of gastric cancer includes host genetic factor,

Correspondence to: Dr Yin Zhu or Dr Nonghua Lu, Department of Gastroenterology, The First Affiliated Hospital of Nanchang University, 17 Yongwai Main Street, Donghu, Nanchang, Jiangxi 330006, P.R. China

E-mail: zhuyin27@sina.com.cn

E-mail: lunonghua@ncu.edu.cn

${ }^{*}$ Contributed equally

Key words: Helicobacter pylori, gastric cancer, hepatocyte growth factor, c-Met for instance eating habits and Helicobacter pylori infection. H. pylori is a gram-negative bacterium that infects $50 \%$ of the world's population and is considered the primary cause of peptic ulcer and chronic gastritis (2). In 1994, the International Agency for Research of Cancer defined H. pylori as a class I carcinogen of gastric cancer which serves an important function in the early stage of gastric carcinogenesis (2). Furthermore, patients with gastric cancer with $H$. pylori infection may exhibit a poor prognosis (3); however, the underlying molecular mechanism of $H$. pylori infection leading to gastric cancer remains unknown.

The hepatocyte growth factor (HGF)/c-Met signaling pathway was considered to be associated with the biological development, regeneration and malignancy of tumors. HGF, also known as scatter factor, may regulate cell viability, migration and angiogenesis by binding to its only high-affinity receptor, c-Met (4). Under normal conditions, HGF-induced c-Met tyrosine kinase activation is regulated in epithelial cells by paracrine ligand delivery (5). In addition, this pathway is constitutively activated in a number of types of malignant tumor including ovarian, lung, colon, breast and gastric cancer (6-9). A previous study revealed that aberrant $\mathrm{HGF} / \mathrm{c}-\mathrm{Met}$ activation occurs in gastric carcinogenesis and a number of antibody or small molecules targeting the HGF/c-Met signaling pathway have been evaluated in a cancer therapy clinical trial (7). However, whether aberrant HGF/c-Met activation is associated with $H$. pylori infection remains unknown. To investigate the function of the HGF/c-Met signaling pathway in the carcinogenesis of $H$. pylori infection, in the present study, human gastric biopsies, following clinical endoscopy or gastrectomy, were selected and gastric epithelial cell lines were co-cultured with $H$. pylori in vitro.

\section{Materials and methods}

Patients. Gastric tissue samples were selected from patients who underwent a gastroduodenoscopy at the First Affiliated Hospital of Nanchang University (Nanchang, China) between January 2011 and January 2013. A total of 160 patients were enrolled in the present study, which included 40 chronic gastritis (CNGS), 40 intestinal metaplasia (IM), 40 dysplasia (Dysp) and 40 gastric cancer (GC) samples. Each group of patients (CNGS, TM, Dysp and GC) contained 20 H. pylori-positive and $20 \mathrm{H}$. pylori-negative samples. There 
was no significant difference in the age and sex distribution between these groups. The present study was approved by the Ethics Committee of the First Affiliated Hospital of Nanchang University and written informed consent was obtained from all patients for participation in the present study.

Detection of H. pylori infection. An 'in-house' rapid urease test (RUT) and modified Giemsa staining were employed to determine $H$. pylori infection. The effectiveness of RUT was $>95 \%$ (data not shown). The modified Giemsa staining was carried out in a double-blind fashion. The tissues used for this staining analysis were fixed in $10 \%$ formaldehyde in $\mathrm{Ca}^{2+}$ and $\mathrm{Mg}^{2+}$-free PBS overnight at $4{ }^{\circ} \mathrm{C}$ prior to paraffin embedding. Paraffin-embedded sections of $4 \mu \mathrm{m}$ were cut with a microtome and stored at room temperature. The specimens were then stained with $20 \%$ Giemsa stain for $20 \mathrm{~min}$ at room temperature. Each specimen was reviewed under the light microscope (magnification, x400). H. pylori infection was diagnosed as positive only if the two methods produced positive results and a $H$.pylori-negative diagnosis was classified if the two methods yielded negative results.

Histological examinations. All biopsies from patients with distinct gastric lesions were obtained from the gastric antrum and individual lesions from patients. The tissues used for histological analysis were fixed as aforementioned: In $10 \%$ formaldehyde in $\mathrm{Ca}^{2+}$ and $\mathrm{Mg}^{2+}$-free PBS overnight at $4{ }^{\circ} \mathrm{C}$ prior to paraffin embedding. Paraffin-embedded sections of $4 \mu \mathrm{m}$ were cut with a microtome and stored at room temperature. Pathological diagnosis and classification were carried out according to the criteria of the World Health Organization and the updated Sydney system (10).

Immunohistochemistry (IHC). Paraffin sections were prepared from the gastric tissues or biopsy specimens. For immunohistochemistry, tissue sections were first de-waxed in xylene and sequentially dehydrated in 100, 95 and $85 \%$ ethanol. The sections were immunostained using the PV-9000 Polymer Detection System (Origene Technologies, Inc., Beijing, China) according to the standard staining protocol. Sections were first washed in PBS and endogenous peroxidase was blocked using $3 \% \mathrm{H}_{2} \mathrm{O}_{2}$. Subsequently, the specimens were incubated with the primary antibody overnight at $4^{\circ} \mathrm{C}$. Primary antibodies used were a rabbit polyclonal anti-human HGF (cat. no. ab83760; dilution, 1:400) or a rabbit monoclonal anti-human c-Met (cat. no. ab51067; dilution, 1:800), and were purchased from Abcam (Cambridge, UK). Specimens were washed with PBS, followed by incubation with polymer helper for $30 \mathrm{~min}$ and polyperoxidase-conjugated anti-rabbit immunoglobulin $\mathrm{G}$ (IgG; ready-to-use; PV-9000 Detection System; Origene Technologies, Inc.) for $30 \mathrm{~min}$. Subsequently, specimens were washed three times with PBS and incubated with 3,3-diaminobenzidin (OriGene Technologies) for developing the positive color. The negative control sections were incubated with PBS only, without primary antibodies. The sections were counterstained with hematoxylin and mounted with coverslips.

Review and scoring of immunostained sections. The stained sections were reviewed and scored under a light microscope by two pathologists, without knowing the clinicopathological data. The concordance rates were typically high and data with any grading discrepancies were re-reviewed to finalize the score. Epithelial cells stained yellow or brown in the nucleus and/or cytoplasm were defined as positively stained. Each section was selected, reviewed and scored between five randomly selected fields (magnification, x200). The H-score system was used to evaluate the expression of HGF or c-Met. Combining staining intensity (score, between 0 and 4) with the proportion of positive cells (score, between 0 and 100\%). Each intensity level was multiplied by the proportion of positive cells and all values were summed to obtain the final IHC score (range, between 0 and 400). Scores between 0 and 200 were considered to be associated with negative expression, whereas scores between 201 and 400 were considered to demonstrate positive expression (11).

Cell lines and $H$. pylori strains. The immortalized gastric epithelial mucosa cell line GES-1, established by the Beijing Institute for Cancer Research (Beijing, China), was a kind gift from Professor Y. Ke of Beijing Institute for Cancer Research, and the human gastric cancer AGS cell line were kind gifts from Professor D.M. Fan of Xijing Hospital (Xi'an, China). These two cell lines were cultured in Dulbecco's modified Eagle's medium (DMEM; Hyclone; GE Healthcare, Logan, UT, USA), supplemented with $10 \%$ fetal bovine serum, $100 \mu \mathrm{g} / \mathrm{ml}$ penicillin and $100 \mu \mathrm{g} / \mathrm{ml}$ streptomycin, at $37^{\circ} \mathrm{C}$ in an atmosphere containing $5 \% \mathrm{CO}_{2}$. The cytotoxin-associated gene $\mathrm{A}\left(\mathrm{CagA}^{+}\right)$and $\mathrm{VacA}^{+} H$. pylori type strain ATCC43504 was cultured on Campylobacter agar plates (Diarrheal Disease Research Center, Shanghai, China), containing 10\% sheep blood, and incubated at $37^{\circ} \mathrm{C}$ under microaerophilic conditions for $24 \mathrm{~h}$. The bacteria were suspended in DMEM. The density was estimated using a spectrophotometer $\left(\mathrm{A}_{660}\right)$. GES-1 and AGS cells $\left(2.5 \times 10^{6}\right.$ cells/flask, $25 \mathrm{~cm}^{2}$ flask $)$ were incubated at $37^{\circ} \mathrm{C}$ in $5 \% \mathrm{CO}_{2}$ with $H$. pylori (multiplicity of infection, 50).

Protein extraction and western blot analysis. At exponential growth phase, $\sim 5 \times 10^{6}$ cells were selected and lysed in a buffer containing $0.5 \%$ Lubrol-PX, $50 \mathrm{mM} \mathrm{KCl,} 2 \mathrm{mM} \mathrm{CaCl}_{2}$, $20 \%$ glycerol, $50 \mathrm{mM}$ Tris- $\mathrm{HCl}$ (pH 7.4), $0.1 \%$ protease and $1 \%$ phosphatase inhibitors (Sigma-Aldrich; Merck KGaA, Darmstadt, Germany). The concentrations of protein samples were determined using the bicinchoninic acid assay (Pierce; Thermo Fisher Scientific, Inc., Waltham, MA, USA). Protein $(50 \mu \mathrm{g})$ was separated by SDS-PAGE (10\% gel), with 4X sample buffer $(250 \mathrm{mM}$ Tris-HCl, pH 6.8, 4\% SDS, 10\% glycerol, $0.006 \%$ bromophenol blue and $2 \%$ 2-mercaptoethanol), and denatured by boiling for $5 \mathrm{~min}$ in a water bath. Subsequently, proteins were transferred onto nitrocellulose membranes (Whatman GmbH, Dassel, Germany) and blocked with 5\% non-fat milk in Tris-buffered saline (TBS) containing $0.1 \%$ Tween-20 (TBST). The membranes were incubated overnight with the primary antibody at $4^{\circ} \mathrm{C}$. Primary antibodies used were a rabbit polyclonal anti-human HGF (cat. no. ab83760; dilution, 1:1,000; Abcam) or a rabbit monoclonal anti-human c-Met (cat. no. ab51067; dilution, 1:1,000; Abcam), and a rabbit polyclonal anti-human $\beta$-actin antibody (cat. no. sc-1615-R; dilution, 1:1,000; Santa Cruz Biotechnology, Inc., Dallas, TX, USA) was used as a reference protein. Subsequently, a horseradish peroxidase-conjugated goat anti-rabbit IgG (cat. 
Table I. Differential expression of HGF and C-Met in distinct histological gastric tissue specimens.

\begin{tabular}{|c|c|c|c|c|c|c|c|c|c|}
\hline \multirow[b]{3}{*}{ Group } & \multirow[b]{3}{*}{$\mathrm{n}$} & \multicolumn{8}{|c|}{ Overall score of protein expression } \\
\hline & & \multicolumn{4}{|c|}{ HGF } & \multicolumn{4}{|c|}{ c-Met } \\
\hline & & - & + & $\mathrm{PR}, \%$ & P-value & - & + & $\mathrm{PR}, \%$ & P-value \\
\hline Overall & 160 & 88 & 72 & 45.0 & 0.078 & 105 & 55 & 34.4 & \\
\hline CNGS & 40 & 27 & 13 & 32.5 & & 33 & 7 & 17.5 & \\
\hline IM & 40 & 19 & 21 & 52.5 & & 33 & 7 & 17.5 & \\
\hline Dysp & 40 & 25 & 15 & 37.5 & & 28 & 12 & 30.0 & \\
\hline $\mathrm{GC}$ & 40 & 17 & 23 & 57.5 & & 11 & 29 & 72.5 & $<0.001^{\mathrm{a}, \mathrm{b}, \mathrm{c}}$ \\
\hline H. pylori ${ }^{+}$ & 80 & 34 & 46 & 57.5 & & 44 & 36 & 45.0 & \\
\hline CNGS & 20 & 12 & 8 & 40.0 & & 16 & 4 & 20.0 & \\
\hline IM & 20 & 8 & 12 & 60.0 & & 15 & 5 & 25.0 & \\
\hline Dysp & 20 & 9 & 11 & 55.0 & & 11 & 9 & 45.0 & \\
\hline GC & 20 & 5 & 15 & 75.0 & & 2 & 18 & 90.0 & \\
\hline H. pylori ${ }^{-}$ & 80 & 54 & 26 & 32.5 & & 61 & 19 & 23.8 & \\
\hline CNGS & 20 & 15 & 5 & 25.0 & & 17 & 3 & 15.0 & \\
\hline IM & 20 & 11 & 9 & 45.0 & & 18 & 2 & 10.0 & \\
\hline Dysp & 20 & 16 & 4 & 20.0 & $0.022^{\mathrm{d}}$ & 17 & 3 & 15.0 & $0.038^{\mathrm{d}}$ \\
\hline $\mathrm{GC}$ & 20 & 12 & 8 & 40.0 & $0.025^{\mathrm{e}}$ & 9 & 11 & 55.0 & $0.013^{\mathrm{e}}$ \\
\hline
\end{tabular}

${ }^{\mathrm{a}} \mathrm{vs}$. overall CNGS; ${ }^{\mathrm{b}} \mathrm{vs}$. overall IM; ${ }^{\mathrm{c}} \mathrm{vs}$. overall Dysp; ${ }^{\mathrm{d}} \mathrm{vs}$. H. pylori ${ }^{+}$Dysp; ${ }^{\mathrm{e}} \mathrm{vs}$. H. pylori ${ }^{+}$GC. HGF, hepatocyte growth factor; PR, positive rate; CNGS, chronic gastritis; IM, intestinal metaplasia; Dysp, dysplasia; GC, gastric cancer; +, positive; -, negative.

no. ZB-2301; dilution, 1:10,000; Origene Technologies, Inc.) secondary antibody was incubated with the membrane at $4^{\circ} \mathrm{C}$ for $4 \mathrm{~h}$. The reactions were subjected to incubation with the enhanced chemiluminescence detection system (Pierce; Thermo Fisher Scientific, Inc.) and exposed to X-ray film for visualization. The blots were quantified by Gel-Pro analyzer (version 4.0; Media Cybernetics, Inc., Rockville, MD, USA).

Statistical analysis. Data are presented as the mean \pm standard deviation or as a proportion of the control. The $\chi^{2}$ test (SPSS software, version 16.0 for Windows; SPSS, Inc., Chicago, IL, USA) was performed to evaluate the difference between categorical variables, including sex, among the distinctly defined groups. The one-way analysis of variance test and least significant difference test (SPSS software, version 16.0) was used to determine the differences, including the age of patients in numerical variables among the groups. $\mathrm{P}<0.05$ was considered to indicate a statistically significant difference.

\section{Results}

Expression of HGF and c-Met in distinct gastric lesions, in association with $H$. pylori infection. The expression of c-Met was significantly increased in GC compared with precancerous lesions ( $\mathrm{P}<0.001$; Table I; Fig. 1). The expression of HGF was observed in only $32.5 \%$ of patients with CNGS, whereas between weak and strong expression of HGF was identified in $52.5,37.5$ and $57.5 \%$ of patients with IM, Dysp and GC, respectively, although there was no significant difference in the expression of HGF between the groups (P>0.05; Table I; Fig. 1).
In the presence or absence of $H$. pylori infection, the expression of c-Met was significantly increased in GC samples $(\mathrm{P}<0.001$; Table I; Fig. 1). However, there was no significant difference in the expression of HGF between the groups in the presence or absence of $H$. pylori infection ( $\mathrm{P}>0.05$; Table I; Fig. 1).

The expression of the proteins was compared between $H$. pylori-positive and $H$. pylori-negative patients at distinct pathological stages. In patients with Dysp and GC, the expression of HGF and c-Met was significantly increased in the presence of $H$. pylori infection, compared with that in the absence of the infection $(\mathrm{P}<0.05$; Table I). However, in patients with CNGS and IM, there was no significant difference determined in the expression of HGF and c-Met between patients with and patients without $H$. pylori infection $(\mathrm{P}>0.05$; Table I).

Induced expression of $H G F$ and c-Met by $H$. pylori in non-malignant and malignant gastric cell lines. Non-malignant GES-1 and AGS cells were incubated with H. pylori (multiplicity of infection, 50) and the expression of HGF and c-Met was significantly increased at all time points $(0.5,1,3$ and $6 \mathrm{~h})$, compared with that in the absence of infection ( $\mathrm{P}<0.05$; Fig. 2 ).

\section{Discussion}

The abnormal activation of the HGF/c-Met signaling pathway has been studied in a variety of types of human epithelial cancer. The underlying molecular mechanism includes gene overexpression, focal gene amplification, gene copy-number 


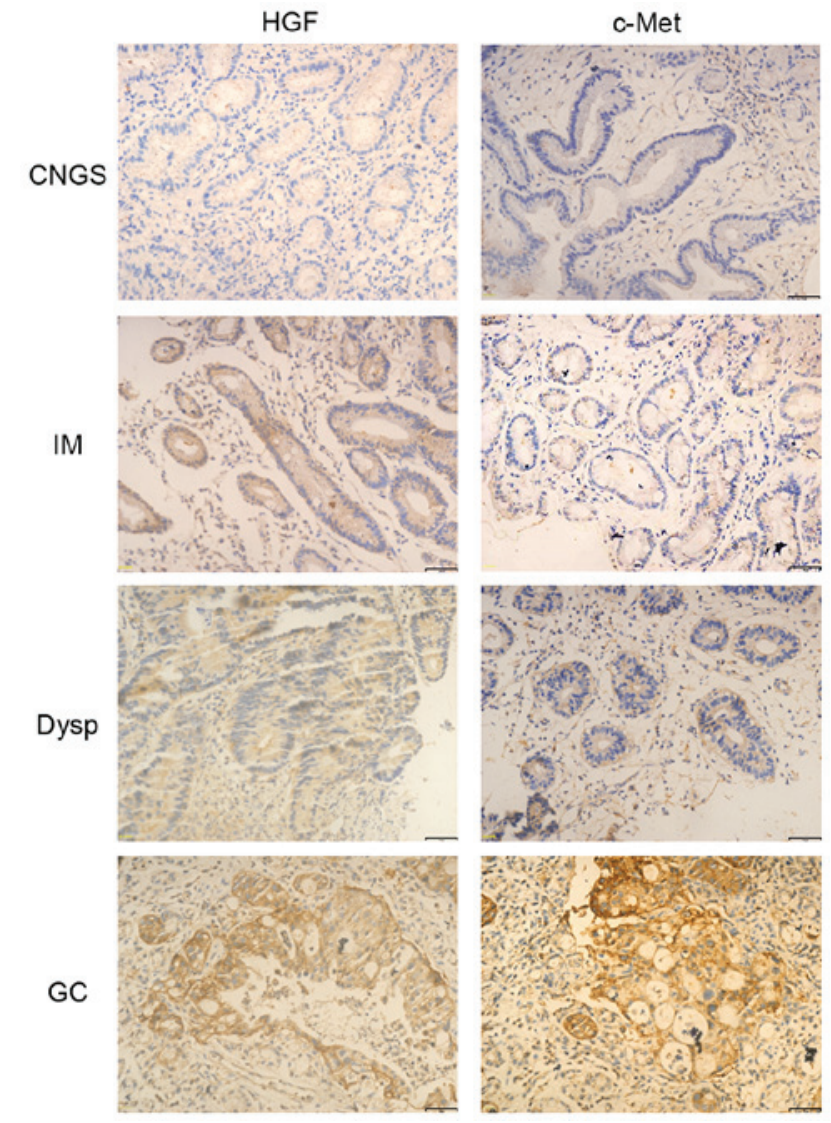

Figure 1. Expression of HGF and c-Met in CNGS, IM, Dysp and GC tissues (magnification, x200; scale bar, $40 \mu \mathrm{m}$ ). HGF, hepatocyte growth factor; CNGS, chronic gastritis; IM, intestinal metaplasia; Dysp, dysplasia; GC, gastric cancer.

gain and activation mutations (11). A previous study revealed that overexpression of c-Met was observed in $46.1 \%$ of gastric carcinoma cases and gene amplification of c-Met was detected in $10.2 \%$ of gastric carcinoma cases (12). Furthermore, the increased expression of c-Met was an indicator of liver metastasis and independent prognostic factors in patients with GC $(12,13)$. The results of the present study identified similar results, as the increased expression of c-Met was observed in the GC group, compared with precancerous lesions. However, no significant difference was identified in the CNGS, IM and Dysp lesions, suggesting that the activation of HGF/c-Met was activated in the later stage of gastric carcinogenesis.

The positive rate of c-Met expression was identified to be increased in the H.pylori-positive Dysp and GC group, compared with that in the $H$. pylori-negative group. The results identified in gastric tissue was validated by the in vitro study. The results of the present study indicated that $H$. pylori infection may activate c-Met expression. The underlying molecular mechanism may be attributed to CagA, which is translocated via a type IV secretion system into epithelial cells, intracellularly modulating the receptor tyrosine kinase c-Met, leading to the activation of extracellular-signal-regulated kinase (ERK) $1 / 2$ in AGS cells (14). Franke et al (15) performed a logical statistical model revealing the differences and commonalities of the response of the network upon HGF and $H$.pylori-induced c-Met signaling, and the results predicted an effect on ERK1/2 signaling induced by the H.pylori stimulus, but not by HGF treatment.
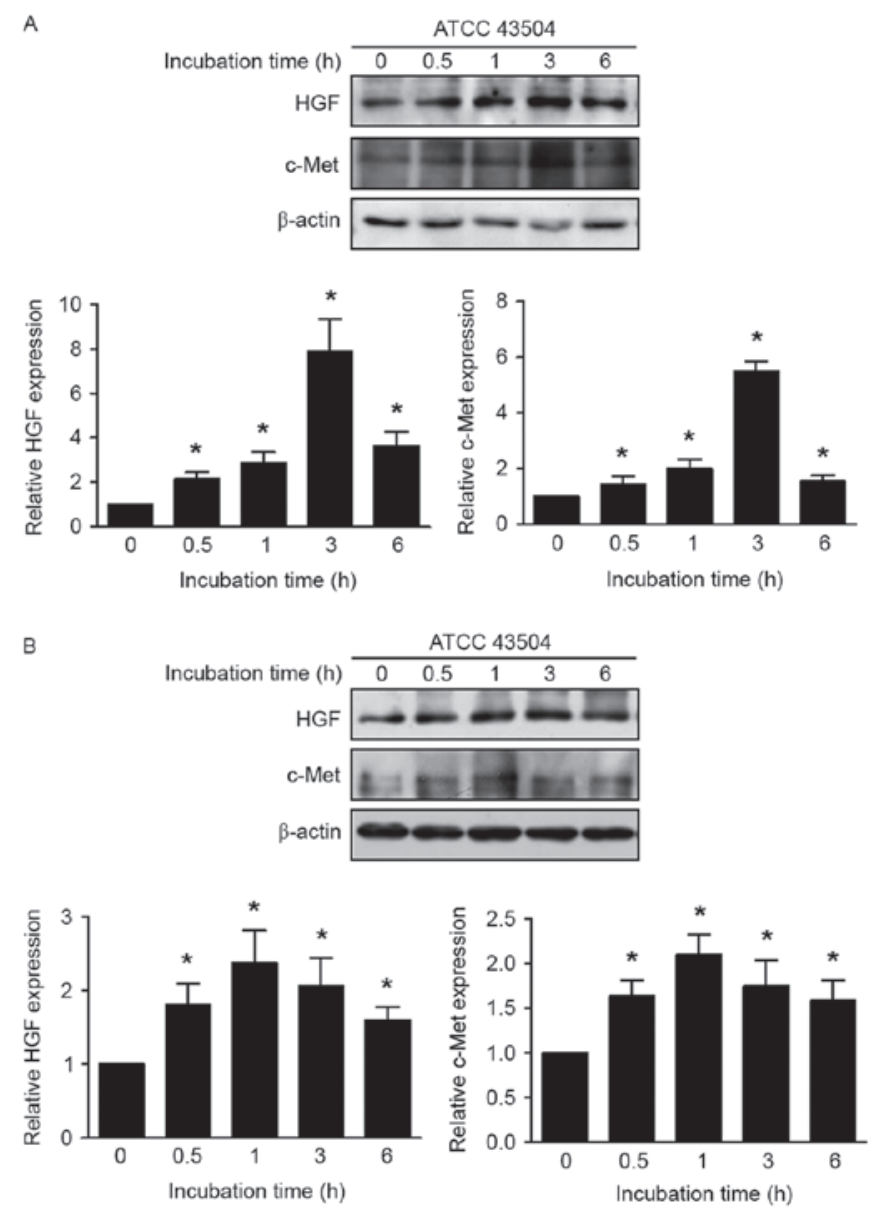

Figure 2. Induction of expression of HGF and c-Met by H.pylori (ATCC43504 strain) in (A) non-malignant GES-1 and (B) malignant AGS gastric cell lines, as determined by western blotting and quantified in the histograms. ${ }^{*} \mathrm{P}<0.05$ vs. Oh group. HGF, hepatocyte growth factor; CNGS, chronic gastritis; IM, intestinal metaplasia; Dysp, dysplasia; GC, gastric cancer.

A previous study identified that the serum HGF levels were markedly increased in the group of patients with gastric cancer and the expression of $\mathrm{HGF}$ was positive in $72 \%$ of GC tissues (16). Whether HGF is an important factor in $H$. pylori pathogenesis remains unknown. The results of the present study demonstrated that the expression of HGF increased in GC samples, compared with that in the precancerous lesions, but with no significant difference. However, the positive rate of HGF was increased in the $H$. pylori-positive Dysp and GC group, which was identified by cytological research in vitro. The controversial results may be due to the complex regulatory mechanisms of HGF in local and systemic physical endocrine systems. In addition, the limitation of gastric samples may have implications for the present study, as it may not comprehensively reflect the level of HGF in entirety. Evaluation of the serum levels of HGF may improve the understanding of the function of HGF in gastric carcinogenesis.

$\mathrm{HGF} / \mathrm{c}-\mathrm{Met}$ activation were identified to be late molecular events in gastric carcinogenesis, which was markedly observed in Dysp and GC with the presence of $H$. pylori infection. $H$. pylori infection may activate the $\mathrm{HGF} / \mathrm{c}-\mathrm{Met}$ signaling pathway in the in vitro study; however, the underlying molecular mechanism remains to be elucidated. 


\section{Acknowledgements}

The present study was supported by the National Natural Science Foundation of China (grant no. 81460116), the Youth Natural Science Foundation of Jiangxi Province (grant no. 20122BAB215009) and the Science and Technology Project of Jiangxi Provincial Education Department (grant no. GJJ10230).

\section{References}

1. Torre LA, Bray F, Siegel RL, Ferlay J, Lortet-Tieulent J and Jemal A: Global cancer statistics, 2012. CA Cancer J Clin 65: 87-108, 2015.

2. Wroblewski LE, Peek RM Jr and Wilson KT: Helicobacter pylori and gastric cancer: Factors that modulate disease risk. Clin Microbiol Rev 23: 713-739, 2010.

3. Li G, Wang Z, Wang Z, Xu J, Cui J, Cai S, Zhan W and He Y: Gastric cancer patients with Helicobacter pylori infection have a poor prognosis. J Surg Oncol 108: 421-426, 2013.

4. Gherardi E and Stoker M: Hepatocyte growth factor-scatter factor: Mitogen, motogen, and met. Cancer Cells 3: 227-232, 1991

5. Prat M, Narsimhan RP, Crepaldi T, Nicotra MR, Natali PG and Comoglio PM: The receptor encoded by the human c-MET oncogene is expressed in hepatocytes, epithelial cells and solid tumors. Int J Cancer 49: 323-328, 1991.

6. Zhou HY, Pon YL and Wong AS: HGF/MET signaling in ovarian cancer. Curr Mol Med 8: 469-480, 2008.

7. Hack SP, Bruey JM and Koeppen H: HGF/MET-directed therapeutics in gastroesophageal cancer: A review of clinical and biomarker development. Oncotarget 5: 2866-2880, 2014.
8. Gelsomino F, Rossi G and Tiseo M: MET and small-cell lung cancer. Cancers (Basel) 6: 2100-2115, 2014.

9. Sipos F and Galamb O: Epithelial-to-mesenchymal and mesenchymal-to-epithelial transitions in the colon. World J Gastroenterol 18: 601-608, 2012.

10. Dixon MF, Genta RM, Yardley JH and Correa P: Classification and grading of gastritis. The updated Sydney System. International Workshop on the Histopathology of Gastritis, Houston 1994. Am J Surg Pathol 20: 1161-1181, 1996.

11. Marano L, Chiari R, Fabozzi A, De Vita F, Boccardi V, Roviello G, Petrioli R, Marrelli D, Roviello F and Patriti A: c-Met targeting in advanced gastric cancer: An open challenge. Cancer Lett 365: 30-36, 2015.

12. Nakajima M, Sawada H, Yamada Y, Watanabe A, Tatsumi M, Yamashita J, Matsuda M, Sakaguchi T, Hirao T and Nakano H: The prognostic significance of amplification and overexpression of c-met and c-erb B-2 in human gastric carcinomas. Cancer 85: 1894-1902, 1999.

13. Amemiya H, Kono K, Itakura J, Tang RF, Takahashi A, An FQ, Kamei S, Iizuka H, Fujii $\mathrm{H}$ and Matsumoto Y: c-Met expression in gastric cancer with liver metastasis. Oncology 63: 286-296, 2002.

14. Churin Y, Al-Ghoul L, Kepp O, Meyer TF, Birchmeier W and Naumann M: Helicobacter pylori CagA protein targets the c-Met receptor and enhances the motogenic response. J Cell Biol 161: 249-255, 2003.

15. Franke R, Muller M, Wundrack N, Gilles ED, Klamt S, Kähne T and Naumann M: Host-pathogen systems biology: Logical modelling of hepatocyte growth factor and Helicobacter pylori induced c-Met signal transduction. BMC Syst Biol 2: 4, 2008.

16. Noguchi E, Saito N, Kobayashi M and Kameoka S: Clinical significance of hepatocyte growth factor/c-Met expression in the assessment of gastric cancer progression. Mol Med Rep 11: 3423-3431, 2015. 